\title{
Sintomatología del síndrome de burnout en deportistas: un estudio con metodología Delphi
}

\section{Athletes burnout symptomatology: a Delphi methodology study \\ Sintomatologia da síndrome de burnout em atleta: um estudo com metodologia Delphi}

\author{
García-Jarillo, Marina (1); De Francisco, Cristina (2); Ortín, Francisco J. (1); Garcés de Los Fayos, \\ Enrique J. (1)
}

(1) Universidad de Murcia, (2) Universidad Católica de Murcia

\section{RESUMEN}

El objetivo de esta investigación fue describir los síntomas conductuales, cognitivos y físiológicos del síndrome de burnout en deportistas, así como conocer si existen diferencias en la percepción del síndrome de burnout entre un grupo de expertos que habían tenido algún deportista con burnout y otro que no lo había tenido. Los participantes del estudio fueron 28 expertos en Psicología del Deporte, 11 mujeres y 17 hombres ( $M$ edad $=46,75$ años; $D T=10,98)$ a quienes se les administró tres cuestionarios semiestructurados Delphi diseñados específicamente por los investigadores. Ambos grupos destacan como síntomas conductuales: falta de adherencia al entrenamiento y bajo rendimiento deportivo; como síntomas cognitivos: desvalorización de la práctica deportiva e ideas de abandono del deporte; y como síntomas fisiológicos: tensión, y agotamiento físico. Con el acercamiento a la sintomatología característica presentada por los deportistas con el síndrome en este estudio se ha pretendido obtener una mejor comprensión del burnout en el ámbito deportivo. En función de esta información, se podrían desarrollar estrategias tanto de prevención como de intervención, dirigidas a prevenir o tratar la principal sintomatología.

Palabras clave: burnout, técnica Delphi, deportista, percepción.

ABSTRACT

The aim of the current research was to increase the knowledge of the behavioural, cognitive and physiological symptoms of burnout in athletes, additionally knowing if there are differences in the perception of burnout syndrome between a group of experts who had had an athlete with burnout and another who did not have it. The study participants were 28 experts in Sports Psychology, 11 women and 17 men $(\mathrm{M}$ age $=46.75$ years; $\mathrm{SD}=10.98)$ being administered three semi-structured Delphi questionnaires specifically designed by researchers. Both groups highlight as behavioural symptoms: lack of training adherence and low sport performance; as cognitive symptoms: sport devaluation and ideas of drop out of sport; and as physiological symptoms: tension and physical exhaustion. With the approach to the characteristic symptomatology presented by athletes with the syndrome in this study, we tried to get a better understanding of burnout in the sports field. Related to this information, both prevention and intervention strategies might be developed, aimed at preventing or treating the main symptomatology.

Keywords: burnout, technique Delphi, athlete, perception.

Correspondence to: Marina, García Jarillo. Dirección Postal: Facultad de

Psicología. Universidad de Murcia. Email: marina.jarillo@gmail.com 


\section{García-Jarillo, M., De Francisco, C., Ortín, F.J., \& Garcés de Los Fayos, E.J.}

\section{RESUMO}

O objetivo desta pesquisa foi aumentar o conhecimento sobre os sintomas comportamentais, cognitivos e fisiológicos da síndrome de burnout em atletas, bem como saber se existem diferenças na percepção da síndrome de burnout entre um grupo de especialistas que teve um atleta com burnout e outro que não teve. Os participantes do estudo foram 28 especialistas em Psicologia do Esporte, 11 mulheres e 17 homens (idade $\mathrm{M}=46,75$ anos; $\mathrm{DP}=10,98$ ) que receberam três questionários Delphi semiestruturados, especificamente projetados por pesquisadores. Ambos os grupos se destacam como sintomas comportamentais: falta de adesão ao treinamento e baixo desempenho atlético; como sintomas cognitivos: desvalorização da prática esportiva e idéias de abandono do esporte; e como sintomas fisiológicos: tensão e exaustão física. Se plantean propuestas de futuro como analizar otras variables relacionadas con el síndrome. Com a abordagem da sintomatologia característica apresentada pelos atletas com a síndrome neste estudo, procuramos obter uma melhor compreensão do burnout no campo esportivo. Com base nesta informação, estratégias de prevenção e intervenção poderiam ser desenvolvidas, visando prevenir ou tratar os principais sintomas. Palavras chave: burnout, técnica Delphi, atletas, percepção.

\section{INTRODUCCIÓN}

El estudio del burnout en el ámbito deportivo presenta una amplia implicación tanto en la salud como en la actividad deportiva de los atletas (Goodger, Gorely, Lavalle y Hardwood, 2007). En cuanto a su prevalencia, De Francisco, Garcés de Los Fayos y Arce (2014) y Olivares, Garcés de los Fayos, Ortín y De Francisco (2018) hallaron que cerca del 4\% de deportistas presentaban burnout, Jiménez, Jara y García (1995) el 6\%, Garcés de Los Fayos (1999) un 7,6\% y, posteriormente, Medina (2001) un 10\%. Gustafsson, Kenttä, Hassmén y Lundqvist (2007) estimaron la prevalencia del burnout en deportistas entre el $1 \%$ y el $9 \%$, así como en diversos estudios la situaron en al menos el 10\% (Dubuc-Charbonneau, Durand-Bush y Forneris, 2014; Gustafsson, 2007; Hodge, Lonsdale y Ng, 2008; Ziemainz, Drescher, Schipfer y Stoll, 2015). Reche, De Francisco y Martínez-Rodríguez (2018), hallaron una prevalencia del $4 \%$ aproximadamente en agotamiento físico/emocional y del $5 \%$ en devaluación de la práctica deportiva y reducida sensación de logro. Se trata, por tanto, de un problema para los deportistas (Garcés de Los Fayos y Medina, 2002) y profesionales que trabajan con los mismos (entrenadores, preparadores físicos, psicólogos del deporte, etc.).

La investigación relacionada con el burnout en deportistas se ha ido incrementando progresivamente a lo largo de los años, sobre todo en lo que a trabajos empíricos se refiere (De Francisco, Arce, Vílchez y Vales, 2016; Gomes, Faria y Vilela, 2017; Goodger et al., 2007; Lundkvist et al., 2018; Marín, Ortín, Garcés de Los Fayos y Tutte, 2013; Reche et al., 2018; Wagstaff, Hings, Larner y Fletcher, 2018). Aunque la definición de burnout de Maslach y
Jackson (1981) es ampliamente aceptada (compuesto por las dimensiones agotamiento emocional, despersonalización y reducida realización personal) no es específica del ámbito del deporte. A lo largo de la historia aparecen autores que definen el síndrome en el ámbito deportivo como un estado de agotamiento físico, emocional y mental que sería la causa de un contacto durante largos periodos de tiempo con situaciones con gran implicación emocional (Pines, Aronson y Kafry, 1981); como la pérdida progresiva de idealismo, energía, motivación y entusiasmo, y por la presencia de fatiga e irritabilidad (Feigley, 1984); como un agotamiento emocional, en una actitud impersonal con el resto de personas, y en la disminución del rendimiento (Fender, 1989); y como agotamiento físico/emocional, devaluación de la práctica deportiva y reducida sensación de logro (Raedeke, 1997).

El síndrome de burnout está formado por componentes físicos, mentales y conductuales (Smith, 1986), los cuales lo definen y lo caracterizan. Los síntomas conductuales que podría presentar un deportista con el síndrome engloban un gran abanico como el descenso en el nivel de eficiencia, aumento de la "retirada psicológica" (cuando no también física), mayor desorganización conductual en los diversos planos vitales, alienación, y disminución de los recursos ambientales por alejamiento y abandono de los mismos (Smith, 1986). Además, intentos de evitar la práctica deportiva o el entrenamiento (Gustafsson, Hassmén, Kenttä y Johansson, 2008), bajo apoyo social de su entrenador o entrenadores (Raedeke y Smith, 2001), entrenadores percibidos rígidos y controladores (Raedeke, 1997), pobre relación entre el 


\section{Sintomatología del síndrome de burnout en deportistas}

deportista y el entrenador (conflictos, pobre comunicación, falta de empatía del entrenador) (Cresswell y Eklund, 2007; Gustafsson et al., 2008) y falta de apoyo social (Cresswell y Eklund, 2004; Cresswell, 2009; Goodger et al., 2007; Raedeke y Smith, 2004).

En cuanto a los síntomas cognitivos, Coakley (1992) alude a un autoconcepto unidimensional referido al autoconcepto deportivo como síntoma favorecedor del desarrollo del síndrome. La cantidad de tiempo empleado por los deportistas en la actividad deportiva limitaría la posibilidad de desarrollar un autoconcepto multidimensional. Al darse una fuerte relación entre la identidad de los deportistas y el deporte, sus logros, emociones e incompetencia influirán negativamente en su autoestima, emociones y motivación (Brewer, Van Raalte y Linder, 1993). Es así que, elevados niveles de burnout se asociarían a una falta de motivación, mientras que altos niveles de motivación intrínseca correlacionan negativamente con el síndrome (Balaguer et al., 2009; Cresswell y Eklund, 2005; Isoard-Gautheur, Guillet-Descas y Lemyre, 2012; Lemyre, Treasure y Roberts, 2006; Raedeke y Smith, 2001).

Entre los síntomas fisiológicos del síndrome de burnout se incluyen el entrenamiento excesivo o sobreentrenamiento, es decir, las cargas de entrenamiento negativas. Dicho sobreentrenamiento también implicaría disfunciones psicofisiológicas, así como no alcanzar las demandas de la competición (Silva, 1990) y/o el incremento de las lesiones deportivas (Cresswell y Eklund, 2006b; DiFiori et al., 2014; Gould, Tuffey, Udry y Loerh, 1996), estando relacionado con la fatiga (Sánchez-Beleña y GarcíaNaveira, 2017). Como síntomas fisiológicos, Smith (1986) describe los siguientes: tensión, fatiga e irritabilidad no acordes con el momento deportivo, dificultad para experimentar emociones positivas como ilusión, expectativas positivas, energía, etc., desórdenes del sueño, mayor susceptibilidad a padecer enfermedades, y tendencia a que desaparezca el entusiasmo en el deporte. Igualmente, abordando la sintomatología de tipo fisiológica, los deportistas más vulnerables a fatigarse serán más propensos a desarrollar burnout (Baumeister, Vohs y Tice, 2007), presentando fatiga crónica (Baumeister et al., 2007; Silva, 1990; Weinberg y Gould, 1999). Y la insuficiente recuperación en la práctica deportiva se manifiesta por la falta de días libres de entrenamiento o de tiempo para dicha recuperación (Gustafsson et al.,
2008). Un síntoma fisiológico del síndrome de burnout, señalado por Coakley (1992) es el estrés o elevados niveles de estrés (Raedeke y Smith, 2004), y no una causa como afirmaban otros autores (Silva, 1990; Smith, 1986).

Por ello, en función de la literatura previa analizada, el objetivo de la presente investigación fue describir los síntomas conductuales, cognitivos y fisiológicos del síndrome de burnout en deportistas, a través de la percepción del psicólogo del deporte mediante el uso de la técnica Delphi. Además, conocer si existen diferencias en la percepción del síndrome de burnout entre un grupo de expertos que habían tenido algún deportista con burnout y otro que no lo había tenido.

\section{MATERIAL Y MÉTODOS}

\section{Participantes}

Participaron en el estudio 28 expertos en Psicología del Deporte (11 mujeres y 17 hombres), con edades comprendidas entre los 24 y los 68 años de edad $(M=46,75 ; D T=10,98)$ y de 19,43 años de experiencia de media $(D T=9,18)$. El método Delphi se realizó a través de correo electrónico, contactando inicialmente con un total de 65 expertos, siendo el porcentaje de rechazos del 43,08\%.

De los expertos, $15(53,6 \%)$ trabajan actualmente como profesor de universidad, $8(28,6 \%)$ como psicólogo del deporte en el ámbito privado, 3 $(10,7 \%)$ como psicólogo en el ámbito privado, y 2 $(7,1 \%)$ son profesores universitarios y psicólogos en la práctica privada.

De la muestra final, $17(60,7 \%)$ habían tenido algún deportista con burnout (grupo 1), y $11(39,3 \%)$ no habían tenido ningún deportista que presentara el síndrome (grupo 2). Todos los expertos aceptaron participar voluntariamente en la investigación, y dieron su consentimiento para utilizar los datos con fines académico-científicos.

El estudio se llevó a cabo de conformidad con la Declaración de Helsinki (WMA 2000, Bošnjak 2001, Tyebkhan 2003), que establece los principios éticos fundamentales para la investigación con seres humanos. Se siguieron las recomendaciones de Buena Práctica Clínica de la CEE (documento 111/3976/88 de julio de 1990).

Instrumentos

El instrumento de evaluación consistió en tres cuestionarios semiestructurados Delphi diseñados específicamente para esta investigación siguiendo una 


\section{García-Jarillo, M., De Francisco, C., Ortín, F.J., \& Garcés de Los Fayos, E.J.}

investigación previa del burnout con esta metodología (García-Jarillo, De Francisco y Garcés de Los Fayos, 2016). En el primer cuestionario (Delphi-1) las preguntas fueron abiertas, mientras que el resto de cuestionarios (Delphi-2 y Delphi-3) tuvieron un formato de respuesta tipo Likert de 5 puntos $(1=$ Muy en desacuerdo; 2 = En desacuerdo; 3 = Neutral; 4 = De acuerdo; y 5 = Muy de acuerdo).

El tercer cuestionario (Delphi-3), del que se presentan los resultados al ser el definitivo, se compone de 3 bloques de preguntas sobre síntomas o manifestaciones de tipo conductual (15 ítems), de tipo cognitivo (24 ítems), y de tipo fisiológico (12 ítems), formado por las opciones de respuesta dadas por los participantes en el primer cuestionario, y valoradas con un mayor acuerdo en el segundo cuestionario. El nivel de consistencia interna de Delphi-3 (Alfa de Cronbach) fue de 0,88, 0,92 y 0,94 para cada uno de los bloques y de 0,96 para la totalidad del cuestionario. Procedimiento

El grupo coordinador del estudio elaboró la primera versión del cuestionario Delphi (Delphi-1), que fue enviado a través de correo electrónico a los expertos junto con una carta de presentación. En ella se explicaban los objetivos, la metodología a seguir, y se les daban instrucciones para cumplimentar Delphi1 y una fecha de respuesta de 3 semanas. Este proceso constituyó la fase preliminar o primera ronda.

La fase exploratoria o segunda ronda se inició con la recepción de los cuestionarios de todos los participantes, con lo que el grupo coordinador analizó las respuestas dadas y diseñó Delphi-2. Este nuevo cuestionario se envió a los expertos, junto con un breve análisis descriptivo del anterior. En este sentido, se dio feedback del grupo coordinador al panel de expertos, analizando posteriormente las respuestas de Delphi-2 y elaborando el tercer cuestionario con la respuesta estadística de grupo.

En la fase final o tercera ronda se envió el último cuestionario a los expertos junto con un análisis descriptivo con la información obtenida en Delphi-2. Por último, se realizó el análisis de Delphi-3 y se enviaron los resultados al panel de expertos a través de un informe final. Dicho informe contiene el nivel de consenso alcanzado por el grupo de expertos. El proceso Delphi completo tuvo una duración total de 16 meses. Se estableció el nivel de consenso al situarse la media de las puntuaciones del total de los expertos entre el intervalo 4-5, indicando que estaban de acuerdo o muy de acuerdo con la variable analizada.

Análisis estadístico

El análisis univariado y bivariado de los datos fue realizado en tres pasos por medio del paquete estadístico SPSS v.22. En primer lugar, se dividió al total de expertos participantes entre aquellos que habían tenido algún deportista con síndrome de burnout $(\mathrm{n}=17)$ y aquellos que no $(\mathrm{n}=11)$, con el fin de analizar si hubieran diferencias estadísticamente significativas en su percepción de la sintomatología, según hubieran tenido algún caso o no. En segundo lugar, se calculó la media muestral y desviación estándar de los diferentes síntomas analizados (conductuales, cognitivos y fisiológicos) para cada grupo de expertos. Finalmente, se calculó la prueba $t$ de Student para muestras independientes, con el fin de observar si había diferencias estadísticamente significativas entre ambos grupos, adoptando un valor de $p \leq 0,05$.

\section{RESULTADOS \\ Síntomas conductuales del burnout en deportistas}

La Tabla 1 muestra la media y desviación estándar de los síntomas conductuales del síndrome de burnout en deportistas para el grupo de expertos que habían tenido algún deportista con burnout y el grupo que no lo habían tenido. En ambos grupos de expertos destacan la falta de adherencia al entrenamiento $(M=$ 4,53, $D T=0,51$ y $M=4,73, D T=0,46$, respectivamente), bajo rendimiento deportivo $(M=$ $4,53, D T=0,62$ y $M=4,36, D T=0,50)$, apatía $(M=$ $4,53, D T=0,51$ y $M=4,45, D T=0,68)$, falta de confianza $(M=4,53, D T=0,51$ y $M=4,45, D T=$ $0,68)$ y fatiga/agotamiento emocional $(M=4,53, D T$ $=0,62$ y $M=4,73, D T=0,46)$ como los principales síntomas conductuales del síndrome de burnout en deportistas, no hallándose diferencias estadísticamente significativas en ninguna de las variables analizadas $(p>0,05)$ de ambos grupos de expertos. 


\section{Sintomatología del síndrome de burnout en deportistas}

Tabla 1

Síntomas conductuales del síndrome de burnout en deportistas

\begin{tabular}{lccccc}
\hline & \multicolumn{2}{c}{$\begin{array}{c}\text { Expertos con } \\
\text { burnout } \\
(\mathbf{n = 1 7 ;} \mathbf{6 0 , 7 \%})\end{array}$} & $\begin{array}{c}\text { Expertos sin } \\
\text { burnout } \\
(\mathbf{n = 1 1 ;} \mathbf{3 9 , 3 \%})\end{array}$ & $\boldsymbol{\boldsymbol { D }}$ \\
\hline Delphi-3 & $\boldsymbol{M}$ & $\boldsymbol{D T}$ & $\boldsymbol{M}$ & $\boldsymbol{D T}$ & $\boldsymbol{t}$ \\
\hline 1. Falta de adherencia entrenamiento & 4,53 & 0,51 & 4,73 & 0,46 & 0,313 \\
2. Conductas de evitación & 4,35 & 0,78 & 4,27 & 0,64 & 0,780 \\
3. Bajo rendimiento deportivo & 4,53 & 0,62 & 4,36 & 0,50 & 0,468 \\
4. Bajo rendimiento académico/laboral & 3,65 & 0,99 & 3,91 & 0,94 & 0,494 \\
5. Escasas/nulas conductas proactivas & 3,82 & 0,80 & 4,09 & 0,70 & 0,377 \\
6. Conflictos con entrenador & 3,82 & 0,72 & 4,09 & 0,70 & 0,344 \\
7. Conflictos familiares & 3,47 & 0,80 & 3,64 & 0,67 & 0,575 \\
8. Conductas inadaptadas & 3,82 & 0,63 & 4,00 & 0,77 & 0,516 \\
9. Cambios en interacciones sociales & 4,00 & 0,61 & 4,27 & 0,46 & 0,220 \\
10. Aislamiento social & 3,76 & 0,56 & 4,09 & 0,70 & 0,185 \\
11. Apatía & 4,53 & 0,51 & 4,45 & 0,68 & 0,744 \\
12. Falta de confianza & 4,53 & 0,51 & 4,27 & 0,64 & 0,254 \\
13. Inestabilidad emocional & 4,41 & 0,61 & 4,64 & 0,50 & 0,324 \\
14. Fatiga/agotamiento emocional & 4,53 & 0,62 & 4,73 & 0,46 & 0,377 \\
15. Falta de empatía & 3,59 & 0,87 & 3,91 & 0,53 & 0,285 \\
\hline
\end{tabular}

\section{Síntomas cognitivos del burnout en deportistas}

Las puntuaciones obtenidas sobre los síntomas cognitivos del síndrome de burnout en deportistas para ambos grupos de expertos se muestran en la Tabla 2. Entre el grupo de expertos que habían tenido algún deportista con burnout, destacan: falta de motivación $(M=4,53, D T=0,62)$, agotamiento cognitivo/mental $(M=4,59, D T=0,50)$, disminución/pérdida del autoconcepto deportivo $(M=$ $4,47, D T=0,62)$, falta de confianza/seguridad $(M=$ $4,47, D T=0,51)$, bajas expectativas de éxito/logro $(M$ $=4,47, D T=0,51)$ e ideas de abandono del deporte $(M$ $=4,47, D T=0,71)$. Por otro lado, los síntomas cognitivos que obtuvieron mayor consenso para el grupo de expertos que no habían tenido ningún deportista con burnout fueron: agotamiento cognitivo/mental $(M=4,73, D T=0,46)$, desvalorización de la práctica deportiva $(M=4,64, D T$ $=0,50)$, disminución/pérdida del autoconcepto deportivo $(M=4,45, D T=0,68)$, ideas de abandono del deporte $(M=4,64, D T=0,50)$ y falta de objetivos $(M=4,45, D T=0,52)$. No se encontraron diferencias estadísticamente significativas entre el grupo 1 y 2 en ninguno de los síntomas cognitivos analizados $(p>$ $0,05)$.

\section{Tabla 2}

Sintomas cognitivos del síndrome de burnout en deportistas

\begin{tabular}{|c|c|c|c|c|c|}
\hline \multirow[b]{2}{*}{ Delphi-3 } & \multicolumn{2}{|c|}{$\begin{array}{c}\text { Expertos con } \\
\text { burnout } \\
(\mathrm{n}=17 ; \mathbf{6 0 0 , 7 \%}) \\
\end{array}$} & \multicolumn{2}{|c|}{$\begin{array}{c}\text { Expertos sin } \\
\text { burnout } \\
(\mathrm{n}=11 ; \mathbf{3 9 , 3 \%})\end{array}$} & \multirow[b]{2}{*}{$t$} \\
\hline & $M$ & $D T$ & $M$ & $D T$ & \\
\hline 1. Falta de motivación & 4,53 & 0,62 & 4,36 & 0,50 & 0,468 \\
\hline 2. Agotamiento cognitivo/mental & 4,59 & 0,50 & 4,73 & 0,46 & 0,472 \\
\hline 3. Falta de concentración & 4,35 & 0,70 & 4,36 & 0,67 & 0,968 \\
\hline 4. Dificultad atencional & 4,41 & 0,71 & 4,36 & 0,67 & 0,860 \\
\hline 5. Problemas de memoria & 3,59 & 0,79 & 4,09 & 0,83 & 0,121 \\
\hline 6. Bloqueo intelectual/mental & 3,76 & 0,83 & 4,27 & 0,78 & 0,119 \\
\hline 7. Desvalorización práctica deportiva & 4,35 & 0,60 & 4,64 & 0,50 & 0,210 \\
\hline
\end{tabular}




\section{García-Jarillo, M., De Francisco, C., Ortín, F.J., \& Garcés de Los Fayos, E.J.}

\begin{tabular}{llllll} 
8. Disminución/pérdida autoconcepto deportivo & 4,47 & 0,62 & 4,45 & 0,68 & 0,950 \\
9. Autodesvalorización & 4,41 & 0,61 & 4,00 & 0,44 & 0,068 \\
10. Baja autoestima & 3,94 & 0,65 & 3,91 & 0,53 & 0,894 \\
11. Disminución/pérdida autoconcepto & 3,94 & 0,65 & 4,00 & 0,44 & 0,798 \\
12. Indefensión & 4,18 & 0,72 & 4,00 & 0,63 & 0,516 \\
13. Desesperanza & 4,00 & 0,79 & 4,09 & 0,53 & 0,741 \\
14. Falta de confianza/inseguridad & 4,47 & 0,51 & 4,09 & 0,53 & 0,073 \\
16. Síntomas depresivos & 3,82 & 0,63 & 4,09 & 0,53 & 0,260 \\
17. Dificultad experimentar emociones positivas & 4,06 & 0,65 & 4,36 & 0,50 & 0,204 \\
18. Pensamientos negativos/catastrofistas & 4,00 & 0,50 & 4,27 & 0,78 & 0,270 \\
19. Pensamientos irracionales & 3,76 & 0,83 & 4,27 & 0,64 & 0,098 \\
20. Preocupación excesiva & 4,00 & 0,61 & 4,36 & 0,67 & 0,152 \\
21. Bajas expectativas de éxito/logro & 4,47 & 0,51 & 4,18 & 0,40 & 0,128 \\
22. Baja tolerancia frustración & 3,88 & 0,85 & 4,09 & 0,53 & 0,480 \\
23. Ideas abandono deporte & 4,47 & 0,71 & 4,64 & 0,50 & 0,512 \\
24. Percepción y juicio distorsionados & 3,76 & 0,83 & 4,27 & 0,64 & 0,098 \\
25. Falta de objetivos & 3,94 & 0,82 & 4,45 & 0,52 & 0,079 \\
\hline
\end{tabular}

$M=4,45, \quad D T=0,52), \quad$ agotamiento físico/fatiga/cansancio $(M=4,71, D T=0,47$ y $M=$ $4,82, D T=0,40)$, desórdenes del sueño $(M=4,29, D T$ $=0,58$ y $M=4,45, D T=0,68)$ e incremento de lesiones deportivas $(M=4,29, D T=0,68$ y $M=4,36$, $D T=0,80)$, no encontrándose diferencias estadísticamente significativas entre ambos grupos de expertos $(p>0,05)$.

En la Tabla 3 se observan los síntomas fisiológicos del síndrome referidos por los participantes. Las puntuaciones que indican un mayor acuerdo para los dos grupos de expertos fueron: Tabla 3

Síntomas fisiológicos del síndrome de burnout en deportistas

\begin{tabular}{|c|c|c|c|c|c|}
\hline \multirow[b]{2}{*}{ Delphi-3 } & \multicolumn{2}{|c|}{$\begin{array}{c}\text { Expertos con } \\
\text { burnout } \\
(\mathrm{n}=17 ; 60,7 \%)\end{array}$} & \multicolumn{2}{|c|}{$\begin{array}{c}\text { Expertos sin } \\
\text { burnout } \\
(\mathrm{n}=11 ; 39,3 \%)\end{array}$} & \multirow[b]{2}{*}{$t$} \\
\hline & $M$ & $D T$ & $\boldsymbol{M}$ & $D T$ & \\
\hline 1. Tensión & 4,41 & 0,71 & 4,36 & 0,80 & 0,870 \\
\hline 2. Irritabilidad & 4,24 & 0,75 & 4,45 & 0,52 & 0,408 \\
\hline 3. Agotamiento físico/fatiga/cansancio & 4,71 & 0,47 & 4,82 & 0,40 & 0,521 \\
\hline 4. Tendencia padecer enfermedades & 4,06 & 0,89 & 4,09 & 0,70 & 0,921 \\
\hline 5. Desórdenes del sueño & 4,29 & 0,58 & 4,45 & 0,68 & 0,515 \\
\hline 6. Falta/exceso apetito & 4,18 & 0,80 & 4,09 & 0,94 & 0,800 \\
\hline 7. Estrés competitivo elevado & 4,29 & 0,68 & 4,27 & 1,27 & 0,954 \\
\hline 8. Nerviosismo (sudoración, temblores) & 3,88 & 0,78 & 4,18 & 0,75 & 0,324 \\
\hline 9. Quejas psicosomáticas & 4,29 & 0,58 & 4,27 & 0,78 & 0,935 \\
\hline 10. Incremento lesiones deportivas & 4,29 & 0,68 & 4,36 & 0,80 & 0,809 \\
\hline 11. Disfunciones sexuales & 3,59 & 0,79 & 3,55 & 0,93 & 0,898 \\
\hline 12. Dificultad recuperación actividad física & 4,47 & 0,51 & 4,36 & 0,67 & 0,638 \\
\hline
\end{tabular}

\section{DISCUSIÓN Y CONCLUSIONES}

La presente investigación se realizó con el propósito de lograr un mayor acercamiento a la sintomatología del burnout en deportistas y, de esta forma, comprobar si los síntomas establecidos en los trabajos teóricos se corresponden con la descrita por 


\section{Sintomatología del síndrome de burnout en deportistas}

los psicólogos del deporte. Para ello, se empleó el método Delphi.

En este trabajo, además, se analiza si existen diferencias entre la percepción de los psicólogos del deporte que cuentan con experiencia en burnout y los que no. De forma más específica, se ha evaluado la percepción de los expertos en relación a los síntomas conductuales, cognitivos y fisiológicos de dicho síndrome. El hecho de que no haya habido diferencias estadísticamente significativas entre ambos grupos de expertos, parece indicar que la experiencia en burnout de los psicólogos del deporte no es una variable que influya en su percepción del síndrome.

En relación a la sintomatología conductual, los expertos afirmaron que la fatiga o el agotamiento emocional sería uno de los síntomas del síndrome en deportistas, en convergencia con otros estudios (Fender, 1989; Pines et al., 1981; Raedeke, 1997), así como la falta de adherencia al entrenamiento (Gustafsson et al., 2008), bajo rendimiento deportivo (Fender, 1989; Gustafsson et al., 2008; Smith, 1986) y bajo apoyo social (Cresswell y Eklund, 2004; Cresswell, 2009; Goodger et al., 2007; MartínezAlvarado, Guillén y Feltz, 2016; Raedeke y Smith, 2004). Es destacable la figura del entrenador y su relación con el deportista, al ser los conflictos con el entrenador uno de los síntomas referidos por los expertos y apoyado por investigaciones previas (Cresswell y Eklund, 2007; Gustafsson et al., 2008; Raedeke, 1997; Raedeke y Smith, 2001).

Por otro lado, haciendo mención a los síntomas cognitivos, la desvalorización de la práctica deportiva, referida por los expertos, sería equivalente a la dimensión del burnout en deportistas "devaluación de la práctica deportiva" planteado por Raedeke (1997). Las bajas expectativas de éxito o logro muestran relación con la reducida sensación de logro, como dimensión establecida del síndrome. Al igual que ocurre con el agotamiento emocional, la devaluación de la práctica deportiva y la reducida sensación de logro son las dimensiones clásicas atribuidas al burnout en el ámbito deportivo.

Igualmente, como sintomatología del síndrome ha sido identificada por los expertos la falta de motivación, al igual que otros estudios muestran la correlación negativa entre la motivación y el burnout en deportistas (Balaguer et al., 2009; Cresswell y Eklund, 2005; Isoard-Gautheur et al., 2012; Lemyre et al., 2006; Raedeke y Smith, 2001). La disminución o pérdida del autoconcepto muestra apoyo con el autoconcepto unidimensional referido al deporte planteado por Coakley (1992). Es posible que los deportistas, al formarse su autoconcepto mayoritariamente en relación al deporte, su autoconcepto no relacionado con el mismo se vea disminuido o afectado. Otro síntoma cognitivo es la dificultad para experimentar emociones positivas, propuesta por los expertos y, que fue planteada por Smith (1986), quien afirmaba que los deportistas tenían dificultades en experimentar ilusión, expectativas positivas, etc. Además, los expertos señalaron las ideas de abandono del deporte.

En cuanto a los síntomas fisiológicos, es destacable el agotamiento físico o fatiga referido por los participantes en la línea de lo planteado por Feigley (1984), Raedeke (1997) y Smith (1986), así como la tensión, por este último autor. El agotamiento físico junto con el agotamiento emocional son subcategorías pertenecientes ambas al agotamiento, definiendo sólidamente el burnout en deportistas. El estrés competitivo elevado, apoyado por los expertos en Psicología del Deporte en relación a la sintomatología de burnout, encontraría relación con los elevados niveles de estrés evidenciados por Coakley (1992) y, posteriormente, por Raedeke y Smith (2004). El constructo estrés es interesante en el ámbito del deporte, siendo investigado en relación con el burnout en diversos estudios (De Francisco et al., 2016; Kallus y Kellman, 2000; Loew, Marsh y Watson, 2014). También los desórdenes del sueño se incluyen entre la posible sintomatología del burnout según los expertos, apoyado por Smith (1986), haciendo alusión al insomnio.

La tendencia a padecer enfermedades es otro de los síntomas que han expuesto diferentes autores (Cresswell y Eklund, 2006b; Gould et al., 1996), quienes citaron los problemas físicos relacionados con el síndrome. Por su parte, Smith (1986) planteó la mayor susceptibilidad a padecer enfermedades. Dicha tendencia o susceptibilidad se podría relacionar con el incremento de lesiones deportistas (DiFiori et al., 2014; Cresswell y Eklund, 2006b; Gould et al., 1996). Por último, la dificultad de recuperación de la actividad física muestra acuerdo entre los expertos, al igual que lo evidencia Gustafsson y colaboradores (2008) con la insuficiente recuperación en la práctica deportiva. La deficiente recuperación del deportista podría relacionarse igualmente con el síndrome de sobreentrenamiento, pero el burnout se trata de un 


\section{García-Jarillo, M., De Francisco, C., Ortín, F.J., \& Garcés de Los Fayos, E.J.}

síndrome que engloba una mayor sintomatología asociada.

En relación a los resultados concretos del presente estudio, la falta de adherencia al entrenamiento, como síntoma conductual del síndrome de burnout, se refiere a los intentos por parte del deportista de evitar la práctica deportiva o el entrenamiento (Gustafsson et al., 2008). Dicho síntoma se podría relacionar con las conductas de evitación propuestas por los expertos, debido a que la propia evitación va ligada a la falta de adherencia al entrenamiento. Por otro lado, resulta coherente que se produzca un bajo rendimiento en el deporte (Fender, 1989); Gustafsson et al., 2008; Smith, 1986) teniendo en cuenta el resto de sintomatología que experimentaría un deportista con burnout (agotamiento emocional, conductas de evitación, desvalorización de la práctica deportiva, falta de motivación, agotamiento físico, y dificultad en la recuperación de la actividad física, entre otros).

El bajo apoyo social podría asociarse con los cambios en las interacciones sociales que plantearon los expertos. Tanto el bajo apoyo social como los conflictos con el entrenador podrían reflejar la problemática interpersonal que puede manifestarse como otra de las subcategorías del síndrome. Por último, la retirada deportiva se trata de la consecuencia más radical, y de la que existe gran solidez científica (Carlin y Garcés de los Fayos, 2010; Coakley, 1992; Silva, 1990; Smith, 1986), y por este motivo es probable que los psicólogos del deporte identificaran las ideas de abandono del deporte como síntomas cognitivos.

Entre las limitaciones del presente estudio se encuentran, entre otras, que únicamente se muestra la información relativa a la sintomatología del burnout en deportistas, siendo también importante analizar las variables predictoras o las consecuencias del burnout. Además, se muestran los resultados del cuestionario final, aunque también sería interesante mostrar el análisis de las respuestas dadas en las distintas fases del método, ya que de esta forma es posible analizar el proceso en su totalidad. Como propuestas de futuro podríamos citar el analizar otras variables relacionadas con el síndrome de burnout en deportistas como las variables predictoras y moduladoras, tal y como se han abordado en estudios cuantitativos (Salgado, Rivas y García, 2011; Ziemainz et al., 2015); así como realizar estudios longitudinales, a pesar de su dificultad, que nos aporten una mejor comprensión del devenir del burnout; y desarrollar nuevos trabajos de índole cualitativo que, como demuestran Cresswell y Eklund (2006a), permiten acceder a información relevante para la comprensión del burnout, difícil de adquirir de otra manera.

\section{APLICACIONES PRÁCTICAS}

La importancia de identificar los componentes fisiológicos, cognitivos y conductuales y, por tanto, de detectar la sintomatología característica a nivel conductual, cognitivo y fisiológico, radica en la posibilidad de una rápida intervención de los deportistas que puedan presentar el síndrome. Además, el conocimiento y la identificación de los síntomas posibilitarían la puesta en marcha de programas de prevención para el colectivo de deportistas.

Los resultados obtenidos pueden ser de interés tanto para entrenadores, preparadores físicos, psicólogos del deporte, etc., como para los propios deportistas, pudiendo reducirse o eliminarse la sintomatología relacionada con el síndrome de burnout (síntomas conductuales, cognitivos $\mathrm{y}$ fisiológicos).

Con el acercamiento a la sintomatología característica presentada por los deportistas con el síndrome en este estudio se ha pretendido obtener una mejor comprensión del burnout en el ámbito deportivo. En función de esta información, se podrían desarrollar estrategias tanto de prevención como de intervención, dirigidas a prevenir o tratar la principal sintomatología.

\section{REFERENCIAS}

1. Balaguer, I., Duda, J., Castillo, I., Moreno, Y. y Crespo, M. (2009). Interacciones entre las perspectivas situacionales y disposicionales de meta y el burnout psicológico de los tenistas junior de la élite internacional. Acción Psicológica, 6(2), 63-75. http://dx.doi.org/10.5944/ap.6.2.222

2. Baumeister, R. F., Vohs, K. D. y Tice, D. M. (2007). The strength model of self-control. Current Directions in Psychological Science, 16, 351-355. http://dx.doi.org/10.1111/j.14678721.2007.00534.x

3. Brewer, B. W., Van Raalte, J. y Linder, D. E. (1993). Athletic identity: Hercules's muscles or Achilles heel? International Journal of Sport 


\section{Sintomatología del síndrome de burnout en deportistas}

Psychology, 24, 237-254. http://dx.doi.org/10.1037/t15488-000

4. Carlin, M. y Garcés de los Fayos, E. J. (2010). El síndrome de burnout: evolución histórica desde el contexto laboral al ámbito deportivo. Anales de Psicología, 26(1), 169-180.

5. Coakley, J. J. (1992). Burnout among adolescent athletes: A personal failure or social problem? Sociology of Sport Journal, 9(3), 271-285. http://dx.doi.org/10.1123/ssj.9.3.271

6. Cresswell, S. L. (2009). Possible early signs of athlete burnout: a prospective study. Journal of Science and Medicine in Sport, 12, 393-398. http://dx.doi.org/10.1016/j.jsams.2008.01.009

7. Cresswell, S. L. y Eklund, R. C. (2004). The athlete burnout syndrome: proposed early signs. Journal of Sports Sciences, 7, 67-84. http://dx.doi.org/10.1016/S1440-

2440(04)80267-6

8. Cresswell, S. L. y Eklund, R. C. (2005). Motivation and burnout among top amateur rugby players. Medicine and Science in Sports and Exercise, 37, 469-477. http://dx.doi.org/10.1249/01.MSS.0000155398.7 1387.C2

9. Cresswell, S. L. y Eklund, R. C. (2006a). The nature of player burnout in rugby: Key characteristics and attributions. Journal of Applied Sport Psychology, 18(3), 219-239. http://dx.doi.org/10.1080/10413200600830299

10. Cresswell, S. L. y Eklund, R. C. (2006b). Athlete burnout: Conceptual confusion, current research, and future research directions. En S. Hanton y S. Mellalieu (Eds.), Literature reviews in sport psychology (pp. 91-126). New York: Nova Science.

11. Cresswell, S. L. y Eklund, R. C. (2007). Athlete burnout: A longitudinal qualitative study. The Sport Psychologist, 21(1), 1-20. http://dx.doi.org/10.1123/tsp.21.1.1

12. De Francisco, C., Arce, C., Vílchez, M. P. y Vales, Á. (2016). Antecedentes y consecuencias del burnout en deportistas: estrés percibido y depresión. International Journal of Clinical and Health Psychology, 16(3), 239-246. http://dx.doi.org/10.1016/j.ijchp.2016.04.001
13. De Francisco, C., Garcés de Los Fayos, E. J. y Arce, C. (2014). Burnout en deportistas: Prevalencia del síndrome a través de dos medidas. Cuadernos de Psicología del Deporte, 14(1), 29-38. https://doi.org/10.4321/S157884232014000100004

14. DiFiori, J. P., Benjamin, H. J., Brenner, J. S., Gregory, A., Jayanthi, N., Landry, G. L. y Luke, A. (2014). Overuse injuries and burnout in youth sports: a position statement from the American Medical Society for Sports Medicine. British Journal of Sports Medicine, 48, 287-288. http://dx.doi.org/10.1136/bjsports-2013-093299

15. Dubuc-Charbonneau, N., Durand-Bush, N. y Forneris, T. (2014). Exploring Levels of StudentAthlete Burnout at Two Canadian Universities. Canadian Journal of Higher Education, 44, 135 151.

16. Feigley, D. A. (1984). Psychological burnout in high-level athletes. The Physician and Sports Medicine, 12(10), 109-119. http://dx.doi.org/10.1080/00913847.1984.11701 971

17. Fender, L. K. (1989). Athlete burnout: Potential for research and intervention strategies. The Sport Psychologist, 3(1), 63-71. http://dx.doi.org/10.1123/tsp.3.1.63

18. Garcés de Los Fayos, E. J. (1999). Burnout en deportistas: un estudio de la influencia de las variables de personalidad, sociodemográficas y deportivas en el síndrome. Tesis doctoral no publicada, Universidad de Murcia, Murcia.

19. Garcés de Los Fayos, E. J. y Medina, G. (2002). Principios básicos a aplicar en el desarrollo de programas de intervención y prevención en deportistas con el síndrome de burnout. Propuestas desde una perspectiva transnacional. Revista de Psicología del Deporte, 11(2), 259267.

20. García-Jarillo, M., De Francisco, C. y Garcés de Los Fayos, E. J. (2016). El síndrome de burnout en deportistas: estudio piloto sobre la percepción del psicólogo deportivo a través del método Delphi. Cuadernos de Psicología del Deporte, 16(1), 243-250.

21. Gomes, A., Faria, S. y Vilela, C. (2017). Anxiety and burnout in young athletes: The mediating role 


\section{García-Jarillo, M., De Francisco, C., Ortín, F.J., \& Garcés de Los Fayos, E.J.}

of cognitive appraisal. Scandinavian Journal of Medicine \& Science in Sports, 27(12), 21162126. https://doi.org/10.1111/sms.12841

22. Goodger, K., Gorely, T., Lavallee, D. y Harwood, C. (2007). Burnout in sport: A systematic review. The Sport Psychologist, 21(2), 127-151. http://dx.doi.org/10.1123/tsp.21.2.127

23. Gould, D., Tuffey, S., Udry, E. y Loerh, J. (1996). Burnout in competitive junior tennis players: II. Qualitative Analysis. The Sport Psychologist, 10(4), http://dx.doi.org/10.1123/tsp.10.4.341

24. Gustafsson, H. (2007). Burnout in Competitive and Elite Athletes. Tesis doctoral no publicada, Örebro, Örebro University.

25. Gustafsson, H., Hassmén, P., Kenttä, G. y Johansson, M. (2008). A qualitative analysis of burnout in elite Swedish athletes. Psychology of Sport \& Exercise, 9, 800-816. doi: 10.1016/j.psychsport.2007.11.004

26. Gustafsson, H., Kenttä, G., Hassmén, P. y Lundqvist, C. (2007). Prevalence of burnout in adolescent competitive athletes. The Sport Psychologist, 20, 21-37. http://dx.doi.org/10.1123/tsp.21.1.21

27. Hodge, K., Lonesdale, C. y Ng., J. Y. (2008). Burnout in Elite Rugby: Relationships with Basic Psychological Needs Fulfilment. Journal of Sports Sciences, 26, 835-844. http://dx.doi.org/10.1080/02640410701784525

28. Isoard-Gautheur, S., Guillet-Descas, E. y Lemyre, P. N. (2012). A prospective study of the influence of perceived coaching style on burnout propensity in elite adolescent athletes: using a self-determination theory perspective. The Sport Psychologist, 26, 282-298. http://dx.doi.org/10.1123/tsp.26.2.282

29. Jiménez, G. Jara, P. y García, C. (1995). Variables relevantes en el contexto deportivo y síndrome de burnout. Trabajo presentado en el V Congreso Nacional de Psicología de la Actividad Física y el Deporte. Valencia.

30. Kallus, K.W. y Kellmann, M. (2000). Burnout in athletes and coaches. En Y. L. Hanin (Ed.), Emotions in sport (pp. 209-230). Human Kinetics: Champaign.
31. Lemyre, P.-N., Treasure, D. C. y Roberts, G. C. (2006). Influence of variability in motivation and affect on elite athlete burnout susceptibility. Journal of Sport and Exercise Psychology, 28, 32-48. http://dx.doi.org/10.1123/jsep.28.1.32

32. Loew, S. J., Marsh, N. V. y Watson, K. (2014). Symptoms of Meares-lrlen/Visual Stress Syndrome in subjects diagnosed with Chronic Fatigue Syndrome. International Journal of Clinical and Health Psychology, 14, 87-92. http://dx.doi.org/10.1016/S16972600(14)70041-9

33. Lundkvist, E., Gustafsson, H., Davis, P., Holmström, S., Lemyre, N. e Ivarsson, A. (2018). The temporal relations across burnout dimensions in athletes. Scandinavian Journal of Medicine \& Science in Sports, 28(3), 1215-1226. https://doi.org/10.1111/sms. 13000

34. Marín, E., Ortín, F. J., Garcés de Los Fayos, E. J. y Tutte, V. (2013). Análisis bibliométrico de burnout y optimismo en deporte. Revista Euroamericana de Ciencias del Deporte, 2(1), 77-87.

35. Martínez-Alvarado, J. R., Guillén, F. y Feltz, D. (2016). Athletes' motivational needs regarding burnout and engagement. Revista de Psicología del Deporte, 25(1), 65-71.

36. Maslach, C. y Jackson, S. E. (1981). MBI: Maslach Burnout Inventory. Manual. Palo Alto, CA: Universidad de California, Consulting Psychologists Press. http://dx.doi.org/10.1037/t05190-000

37. Medina, G. (2001). Síndrome de Burnout y Locus de Control en deportistas cubanos de alto rendimiento. (Tesis de Licenciatura no publicada). Universidad de la Habana, La Habana.

38. Olivares, E. M., Garcés de los Fayos, E. J., Ortín, F. y De Francisco, C. M. (2018). Prevalencia de burnout a través de dos medidas y su relación con variables sociodeportivas. Universitas $\begin{array}{lll}\text { Psychologica, } & 17(3), & 1-9 .\end{array}$ https://doi.org/10.11144/Javeriana.upsy173.pbmr

39. Pines, A., Aronson, E. y Kafry, D. (1981). Burnout: From tedium to personal growth. En 


\section{Sintomatología del síndrome de burnout en deportistas}

Cherniss, C. (Ed.). Staff burnout: Job stress in the human services. New York: The Free Press.

40. Raedeke, T. D. (1997). Is athlete burnout more than just stress? A sport commitment perspective. Journal of Sport and Exercise Psychology, 19(4), 396-417. http://dx.doi.org/10.1123/jsep.19.4.396

41. Raedeke, T. D. y Smith, A. L. (2001). Development and preliminary validation of an athlete burnout measure. Journal of Sport \& Exercise Psychology, 23(4), 281-306. http://dx.doi.org/10.1123/jsep.23.4.281

42. Raedeke, T. D. y Smith, A. L. (2004). Coping resources and athlete burnout: an examination of stress mediated and moderation hypotheses. Journal of Sport and Exercise Psychology, 26, 525-541. http://dx.doi.org/10.1123/jsep.26.4.525

43. Reche, C., De Francisco, C. y MartínezRodríguez, A. (2018). Relationship among sociodemographic and sport variables, exercise dependence, and burnout: a preliminary study in athletes. Anales de Psicología, 34(2), 398-404. https://doi.org/10.6018/analesps.34.2.289861

44. Salgado, R., Rivas, C. A. y García, A. (2011). Aparición del Burnout en jugadores de fútbol de primera división profesional de México: Estrategias de intervención. Cuadernos de Psicología del Deporte, 11(2), 184-196.

45. Sánchez-Beleña, F. y García-Naveira, A. (2017). Sobreentrenamiento y deporte desde una perspectiva psicológica: estado de la cuestión. Psicología Aplicada al Deporte y al Ejercicio Físico, 2(2), 1-12. https://doi.org/10.5093/rpadef2017a8

46. Silva, J. M. (1990). An analysis of the training stress syndrome in competitive athletics. The Journal of Applied Sport Psychology, 2, 5-20. https://doi.org/10.1080/10413209008406417

47. Smith, R. E. (1986). Toward a cognitive-affective model of athletic burnout. Journal of Sport Psychology, $\quad 8(1), \quad 36-50$. https://doi.org/10.1123/jsp.8.1.36

48. Wagstaff, C., Hings, R., Larner, R. y Fletcher, D. (2018). Psychological Resilience's Moderation of the Relationship Between the Frequency of Organizational Stressors and Burnout in Athletes and Coaches. The Sport Psychologist, 32(3), 17888. https://doi.org/10.1123/tsp.2016-0068

49. Weinberg, R. y Gould, D. (2007). Foundations of Sport and Exercise Psychology. Champaign, Il: Human Kinetics.

50. Ziemainz, H., Drescher, A., Schipfer, M. y Stoll, O. (2015). An Explorative Study of Possible Demographic Variables, Sports-Related Situational Variables, and Social Variables as Predictors of Athlete Burnout and Its Core Dimensions among German Non-Elite Endurance Athletes. Advances in Physical Education, $\quad 5, \quad 60-69$. https://doi.org/10.4236/ape.2015.51008 